\title{
Yielding behavior of copper nanowire in the presence of vacancies
}

\author{
LIU XiaoMing $^{1 *}$, YANG XiaoBin ${ }^{2} \&$ WEI YueGuang ${ }^{1}$ \\ ${ }^{1}$ The State Key Laboratory of Nonlinear Mechanics, Institute of Mechanics, Chinese Academy of Sciences, Beijing 100190, China; \\ ${ }^{2}$ School of Resources and Safety Engineering, China University of Mining \& Technology, Beijing 100083, China
}

Received February 6, 2012; accepted April 5, 2012; published online April 18, 2012

\begin{abstract}
In the present research, the measurement fluctuations of mechanical properties in nanowires (NWs) are investigated by using the molecular dynamics simulation. The large numbers of simulations are performed to study the yield behaviors of the NWs. The results have shown that the yield behavior of the smaller diameter NW is more sensitive to the presence of vacancies, and the dispersion of the measured mechanical properties for the small scale NW is larger than that for the large scale NW. Present results have also shown that vacancies escape from the bulk to the free surfaces as a result of high stress applied at the small scale systems similar to the dislocation starvation phenomenon observed in the compression test of nano-pillars, and dislocation nucleation induced by surface defect occurs after the vacancy reaches free surface leading to lower yield strength. Moreover, the strong surface vacancy interactions at the nanoscale level are also investigated.
\end{abstract}

nanowire, yield strength, vacancies, size effect

PACS number(s): 61.72.Ji, 61.72.Ss, 62.25.+g, 61.46.-w, 62.20.Fe

Citation: Liu X M, Yang X B, Wei Y G. Yielding behavior of copper nanowire in the presence of vacancies. Sci China-Phys Mech Astron, 2012, 55: 10101017, doi: 10.1007/s11433-012-4742-5

Nanoscale materials and structures have attracted a considerable attention in last two decades due to their unique mechanical properties. Metal nanowires (NWs) are of great scientific and technological importances due to their great role in microengineering researches and applications. They exhibit the strong size effects on mechanical properties, such as Young's modulus and yield strength $[1,2]$. Experimental studies [3] and semi-empirical calculations [4] have revealed the significant increase in the yield strength for metal nanowires, sometimes 20 times larger than the bulk materials. Recent developments in experimental and computational techniques have made it possible to evaluate the mechanical properties of NWs. Marszalek et al. [5] have used the atomic force microscope (AFM) to measure the effect of micro-structure of a gold NW on the plastic behavior during loading process. Their results have displayed some information to explore the plastic deformation mecha-

*Corresponding author (email: xiaomingliu@1nm.imech.ac.cn) nism of the NW. On the other hand, a lot of researchers have studied the mechanical behaviors of NWs and nanotubes under various loading conditions by using the atomistic simulations [6-15]. However, there always exist some difference between simulation results and the experimental results [3] for yield strength of nanowires, although they are manufactured by different methods [16-18]. Greer et al. [17] have investigated the yield strength of microscale wire and showed that the results are dependence on the size, microstructure and manufacturing process. They have deduced that the possible reason for the large deviation variation is high density of imperfections from the manufacturing process. A lot of researchers [19-22] have studied the effects of pre-existing defects and microstructure formations on the nanowire inelasticity behavior, experimentally and theoretically, and most results have displayed that both microstructure formation and confinement play a critical role in the mechanical properties of nanoscale materials, and the yield strength and dynamic response of NW are strongly 
dependent of the defects, such as vacancies. The defects are unstable since "dislocation starvation" occurs, particularly in micro- or nanoscale [17,23]. Sadeghian et al. [24,25] have studied the defect effect on the mechanical behavior of silicon nano-plates, and their results have shown that defects significantly influence the size-dependent elastic behavior, such as Young's modulus. Njeim et al. [26] have studied the effect of vacancies on the nanoindentation behavior and their result has displayed that by increasing the vacancy concentration a decrease in the yielding load is followed from a power law relationship.

The point defect determines the partial dislocation emission in the crystal materials and it also affects the structure of stacking faults and nanotwins [27], which are generated by partial dislocation emission from the grain boundary. Annihilation of initial vacancy on the free surface will produce the surface defect as a result of strong surface stress, which is typical in nanomaterial with large surface to volume ratio. The surface defects lower the yield strength since most of the dislocation initiations start from the free surface or atomically sharp corner. Yang et al. [28] have showed that the surface stress is critical for yield strength of ultra-small NW.

In the present research, yield strength dependence on the presence of vacancies is investigated, and vacancy coalescence in the bulk and starvation at the surface is also studied. By statistically analyzing the simulation results, we try to interpret large fluctuation of yield strength of small NW system. The primary reason may be the strong interaction between the vacancy and the free surface in small scale systems.

\section{Simulation method}

The embedded atom method (EAM) potential for copper developed by Mishin et al. [29] is applied to explore the tensile response of NWs. This potential provides the improved accuracy and reliability based on a large set of experimental and $a b$ initio data.

The NWs were prepared by creating a perfect FCC crystal of copper with lattice constant of $3.615 \AA$, and then vacancies were created by randomly removing number of atoms inside the NWs. All the NWs had a square cross-section and a slenderness ratio $(a / l)$ of $1: 3$, where $a$ is the cross-section size and $l$ is the axial length, as shown in Figure 1. A series of sizes of the NWs ranging from $a=18.1 \AA$ to $a=72.3 \AA$ are considered. The orientations of sides in $x, y$ and $z$ directions are $\langle 100\rangle,\langle 010\rangle$ and $\langle 001\rangle$, respectively.

After initial construction, the wires were relaxed by the conjugate gradient algorithm through local energy minimum. The relaxed structures were used as the initial configurations for simulating uniaxial tension. To reduce the influ-

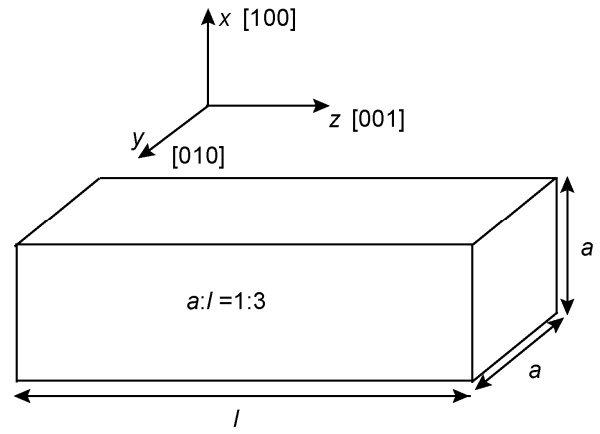

Figure 1 Schematic of the NW size and crystallographic orientations.

ence of thermal fluctuations on nucleation and propagation of defects, we adopted the canonical ensemble via external Nosé-Hoover thermostat $[30,31]$ at a temperature of $1 \mathrm{~K}$. Simulations were performed with free boundary conditions on the side surface of NWs in order to capture the relevant surface effects using an open source code LAMMPS [32]. During the loading process four layers of atoms from left and right ends were displaced at a prescribed uniform strain increment. A uniaxial tensile loading was applied until strain reaching at the $40 \%$ of desired value, the velocity of Verlet algorithm is used at a time step of $1 \mathrm{fs}$. All the tensile simulations in this paper were performed at a strain rate of $\dot{\varepsilon}=8 \times 10^{8} \mathrm{~s}^{-1}$. The tensile stress was calculated by dividing force with sectional area.

In order to visualize defects in the system, we used the local crystalline order analysis, which permits the distinction between the atoms in a local hexagonal close packed (HCP) environment and those in a face-centred cubic (FCC) environment [33]. Atoms were classified with different colours according to their crystalline orders. The transparent atoms with local FCC order represented the perfect FCC lattice, 12 atoms being nearest neighbours with local HCP order are colored with red to represent those in the stacking faults, the remainder of the 12 nearest neighbours are colored with blue. The green atoms with 10 or 11 nearest neighbours represented those in the vacancies, and the yellow atoms with less than 10 nearest neighbours represented disorder [34-37].

\section{Result and discussion}

\subsection{Convergence study of the MD realizations}

The fracture of NW presumes a statistical distribution as suggested by others [38-40], the statistical average of simulations is necessary in the NW system to prevent large fluctuation. More than 4000 simulations are performed on the NW systems with different sizes $a$ and vacancy concentrations $c_{v}$. In each case, with specific size and vacancy concentration we constructed more than 50 configurations with different microscopic structures (locations of vacancy) 
to minimize the effect of spatial distribution of defects. Different concentrations of vacancies in the NWs were created by randomly removing a fraction of atoms. In each case, the vacancy fraction ranges from $0.1 \%$ to $1 \%$, approximately. This range is chosen due to considering the vacancy concentration for most pure metals [41].

At first, we used benchmarks to set up the realization numbers for each set of simulations. The convergence study is shown in Figure 2 for two typical cases, $a=18.1 \AA$ and $a=57.8 \AA$. All the data points were averaged by several realizations ranging from 10 to 100 . It can be seen for both cases that data with 10 realizations gives a scattered result which is inconsistent with the convergent result. It displays the small deviations when the number of average reaches 30 and above. For instance, the difference among 30, 50 and 100 averages shown in Figure 2(a) can be negligible. It also occurs for the difference between 40 and 50 averages, as shown in Figure 2(b). In order to verify the results, we use 100 realizations for smaller NWs, and 50 realizations for a larger NWs. This is because the yielding of smaller NW is more sensitive to defects and needs more statistical averages for obtaining convergence.

\subsection{Vacancy effect on the yield strength}

Simulations for the cases of the various vacancy concentrations and section sizes were performed. We observed the yielding of NW, the first evidence of plastic deformation. The convergent results are collected from more than 4000 computation sets. The relation between the yield load and the vacancy concentration follows a power-law one as suggested in refs. [26,42], and as follows:

$$
\frac{\sigma_{y}}{\sigma_{y 0}}=\left(\frac{c_{v}}{c_{0}}\right)^{-n},
$$

where $\sigma_{y}$ is the yield strength, $c_{v}$ is the vacancy concen-

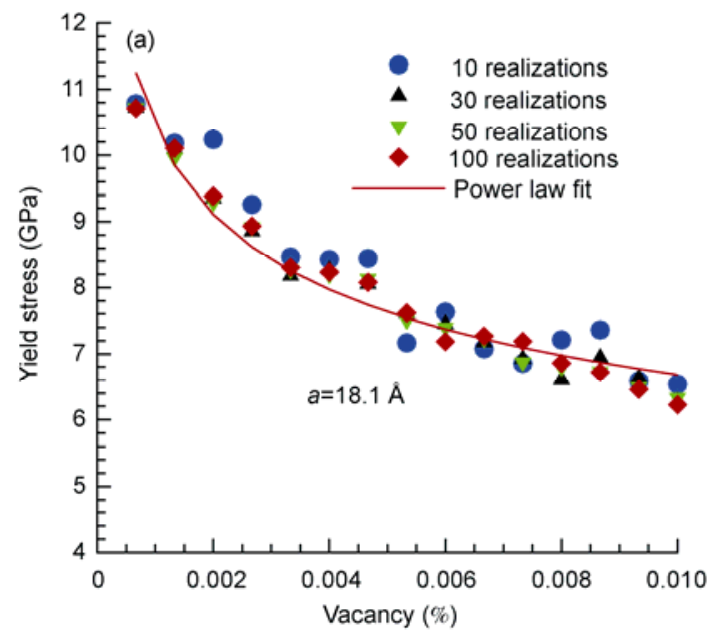

tration, $\sigma_{y 0}$ and $c_{0}$ are the corresponding values at the reference state, here we choose $c_{0}=0.1 \%$ as the reference state value. The power-law index $n$ reflects the variation of yield strength with vacancy concentration.

From Figure 2, the yield stress decrease with increasing vacancy concentration. It indicate that the vacancy lowers the yielding load with the power law and the result is consistent with Huang's result [43]. The power-law index $n$ for each size of NW is shown in Figure 3 and the value ranges from 0.198 to 0.114 , which is consistent with [26], $n=0.083$ for Fe under nano-indentation test [42], $n=0.13$ for bulk copper under dynamic shock wave. The result of the power-law index displays the strong size effect, as shown in Figure 3. It increases with the increase of NW size $a$, meaning that the small size of NW acts more sensitively to the variation of vacancy density. The bigger size has a weaker power-law relation, and the power-law index $n$ for $a=72.3 \AA$ case is approximate $57.5 \%$ of the value of NW with $a=18.1 \AA$. It displays that vacancy concentration is an critical factor in NW with smaller section size.

We also use the following linear model to fit the relationship between yielding stress and vacancy concentrations:

$$
\sigma_{y}-\sigma_{y 0}=k\left(c_{v}-c_{0}\right),
$$

where $k$ is the proportion factor which physically means the linear dependence of yield strength on the vacancy concentration $c_{v}$. The dependence of $k$ on the size of NW is plotted on Figure 4. It shows that the small NW has a high dependence on the $c_{v}$ than a large NWs. The $k$ value for $a=18.1 \AA$ is approximately twice that of $k$ for $a=72.3 \AA$. This simple linear model gives the similar conclusion as the power-law model, thus the physical mechanism of size effect is correctly interpreted by the fitting process in eq. (1).

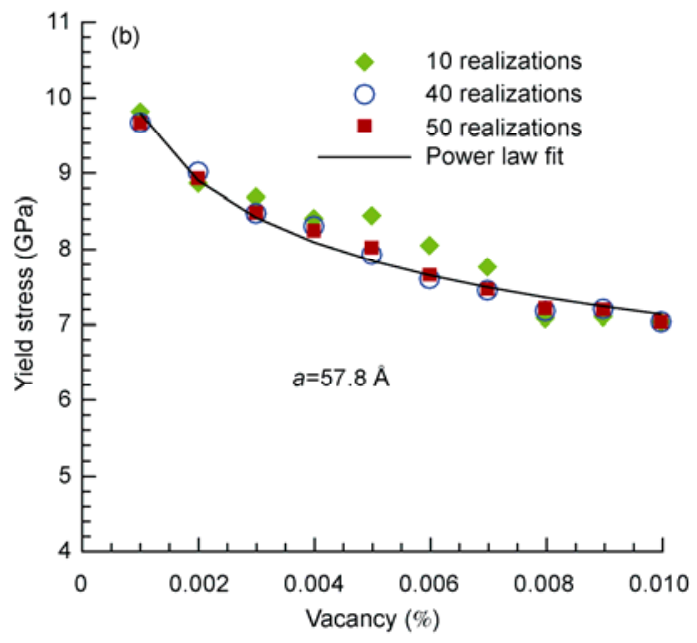

Figure 2 (Color online) Yield strength versus vacancy concentration for different sizes of NW. (a) $a=18.1 \AA$; (b) $a=57.8 \AA$. 


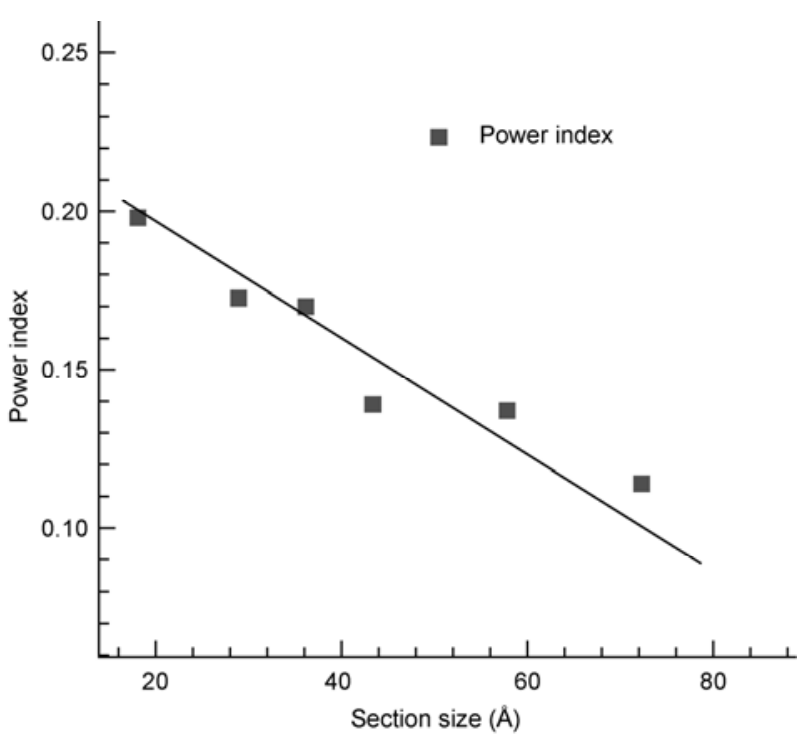

Figure 3 Power index $n$ versus NW size $a$. The solid line in the figure is the linear fit.

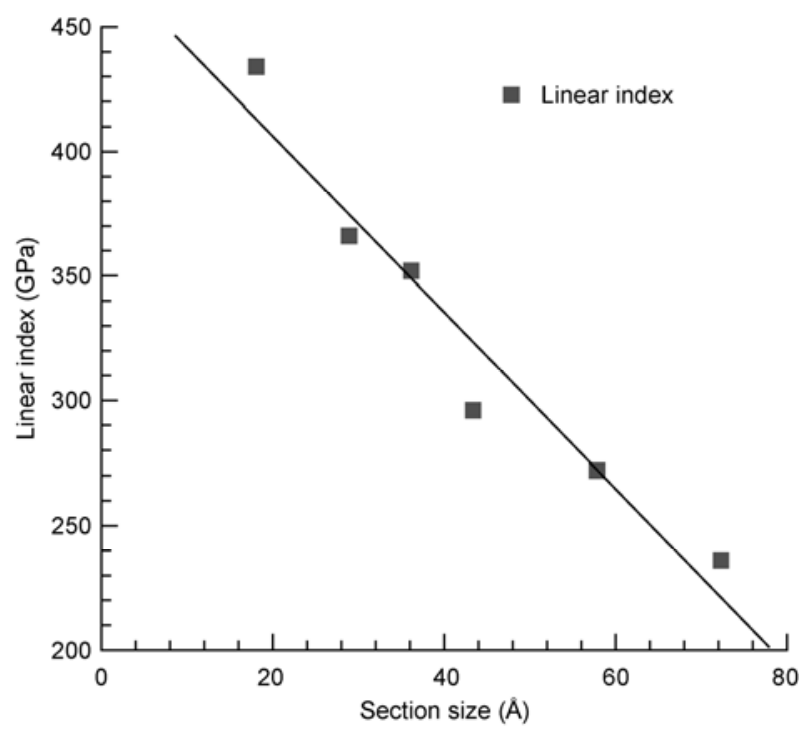

Figure 4 Linear index $k$ versus NW size $a$. The solid line in the figure is the linear fit.

\subsection{Interaction between vacancy and surface}

\subsubsection{Observation of microstructure evolution}

Defects play a critical role in the yield processes, and appear in different formations, such as dislocations and point vacancies. Dislocation is unstable in the NW systems. Dislocation starvation phenomenon is observed in the compression test of nano-pillar. Point defects tend to have the similar phenomenon in the NW. They prefer to immigrate to the free side surface rather than staying in the bulk. The stressstrain relations are analyzed carefully, and the microstructure evolutions are shown in Figures 5-9. The typical stress-strain curve of NW as shown in Figure 5 (size $a=18.1 \AA$ ) and Figure 7 (size $a=72.3 \AA$ ), has one peak at strain of $\sim 1 \%$, and $\sim 0.07 \%$, respectively, followed by several lower peaks and then a decrease [38].In the initial configuration, vacancies exist in the forms of surface defects and point defects as indicated by blue atoms in Figures 6(a) and 8(a) for NWs with different sizes. The large value of the surface to volume ratio in small NW causes most of vacancies which are attracted by the free surfaces.

Surface stress serves an important role in the whole tensile procedure, as indicated by Diao et al. [44]. The smaller the sizes, the higher the surface stress is. In small size case, the phase transformation may occur. With strong surface effect and tensile loading, the point defects migrate to the free surface region in the bulk case, and free surface absorbs defects (Figures 6(a) and 8(a)). This phenomenon is more obvious in small size NW by comparison with that of vacancy fraction, as shown in Figures 5 and 7 (blue curves). In the small NWs case, the vacancy fraction drops to zero before the yield point (in Figure 5), while in large case, the vacancy fraction remains constant (Figure 7). There is not vacancy in small NW case before yield. However, there is large fraction of vacancies in the large NW case. We can also detect the difference by comparison of microstructures, between Figures 6(b) and 8(b). It is notable to view the small jump in the variation of vacancy fraction in Figure 5 (b), which possibly indicates absorption of one vacancy which reaches the surface.

In the small NW case, most portions of the vacancies move to the surface to form surface defects before yield. The surface defects (steps) lower the yield strength because most of the initiation of dislocation starts from the free surface or atomically sharp corners [12]. This may explain why we observe more prominent size effects (Figures 3 and 4). Figure 9 shows the NW surface structure at different strain level as indicated in Figure 5 for ultra small nanowires $(a=1.81 \mathrm{~nm}$.). The surface varies from initial smooth surface as in Figure 9(a) to defected surface as shown in Figure 9(b) corresponding to B point in Figure 7(a) (strain level before yield). Vacancies tend to migrate to the surface, and lead to yield. Figure 9(c) shows the nucleation of defects from resulting surface vacancy which is corresponding to $\mathrm{C}$ point in Figure 7(a). Our findings demonstrate defect starvation in small-volume structures with ultra-small wire geometry, which is governed by a mechanism similar to that of dislocation starvation $[23,45,46]$.

In Figures 6(c) and 8(c), large amounts of stacking fault have formed immediately at the atomically sharp corner or side surface [12] on the one side of the $\{111\}\langle 11 \overline{2}\rangle$ slip systems where the max shear stress is reached. Stacking fault then allows the partial dislocation to propagate along the slip plane. This event apparently leads to yield, which can be determined quantitatively from the abrupt drops in the stress strain curves as shown in Figures 5 and 7. After the burst point, the stacking fault atom portion increased abruptly as shown in stacking fault fraction (Figures 5 and 7). With continued loading, the stacking fault continued 

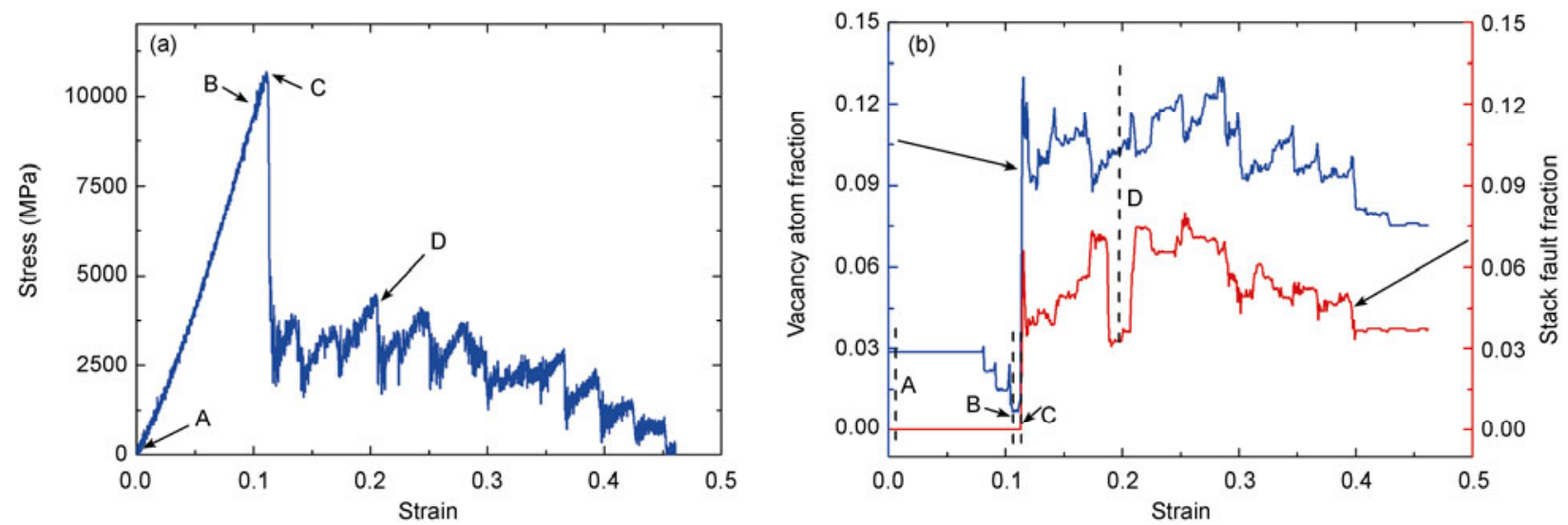

Figure 5 Variation of stress (a) and microstructure (b) as a function of strain in the tensile simulation of the NW of size $a=18.1 \AA$ and initial vacancy fraction $f=0.65 \%$.

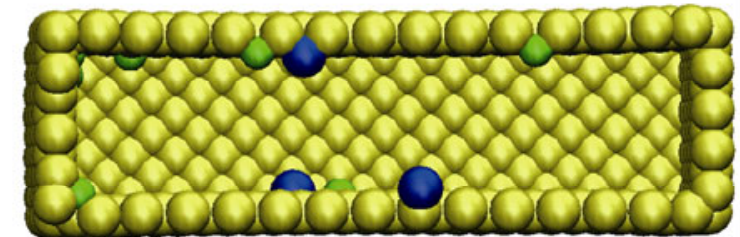

(a)

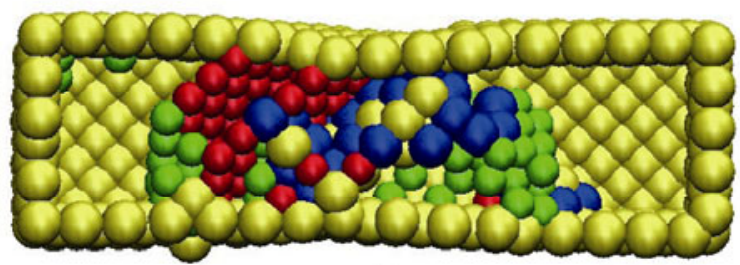

(c)

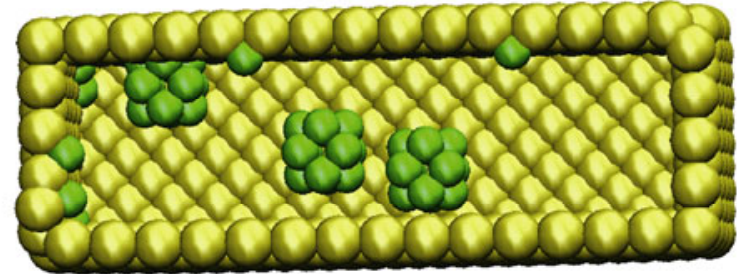

(b)

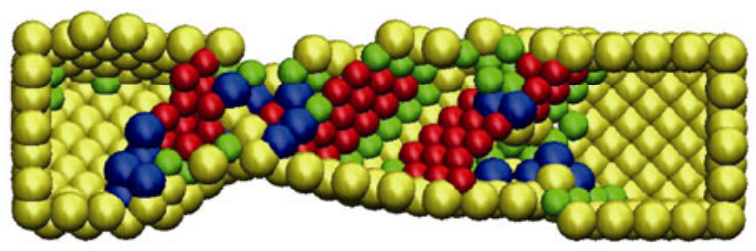

(d)

Figure 6 Atomic structures corresponding to various strain levels indicated in Figure 5.
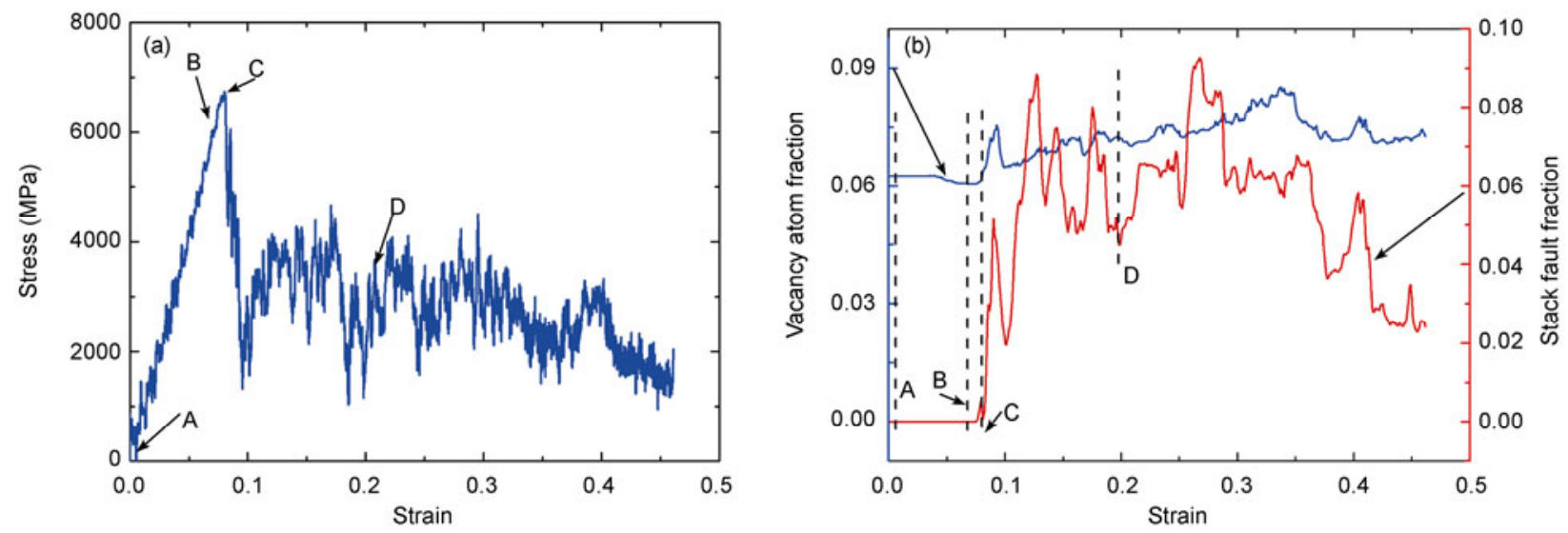

Figure 7 Variation of stress (a) and microstructure (b) as a function of strain in the tensile simulation of the NW of size $a=72.3 \AA$ and initial vacancy fraction $f=0.65 \%$.

nucleation, and the fraction keeps in the level of approximately $0.7 \%$. Partial dislocation at each edge of one stacking fault plane intersected with the free surface in both cases as shown in Figures 6(d) and 8(d).

\subsubsection{Quantitative analysis}

From the NW or nanopillar experiments [3,23], the yield strength has a very large variation over different scales. It has been clearly indicated from experiment that the large 


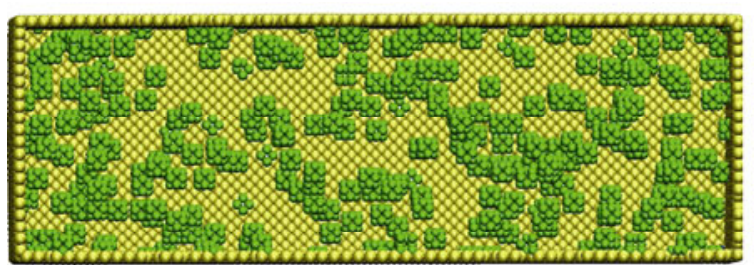

(a)

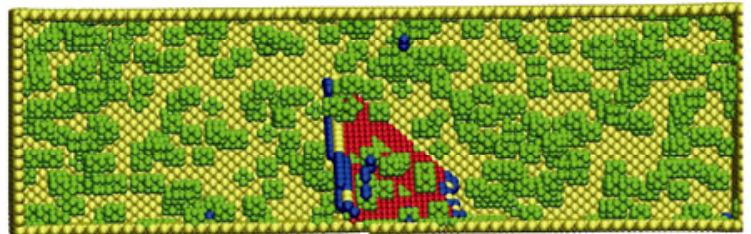

(c)

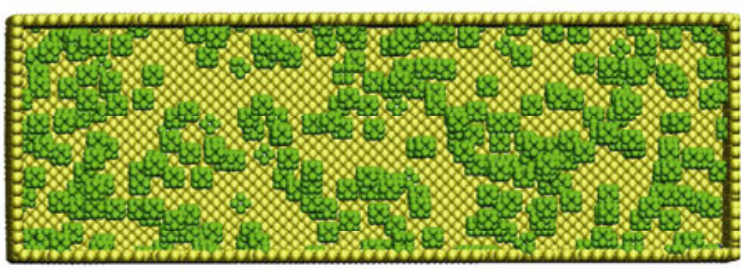

(b)

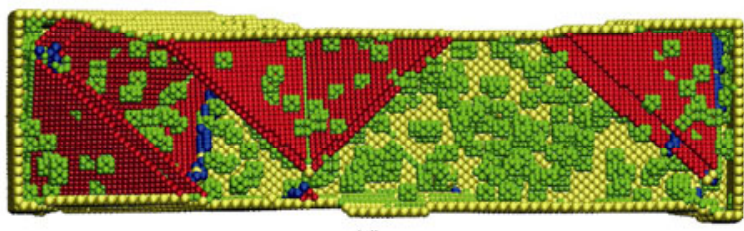

(d)

Figure 8 Atomic structures corresponding to various strain levels as indicated in Figure 7.

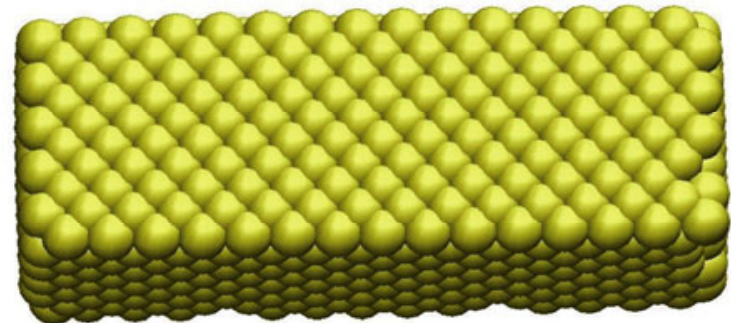

(a)

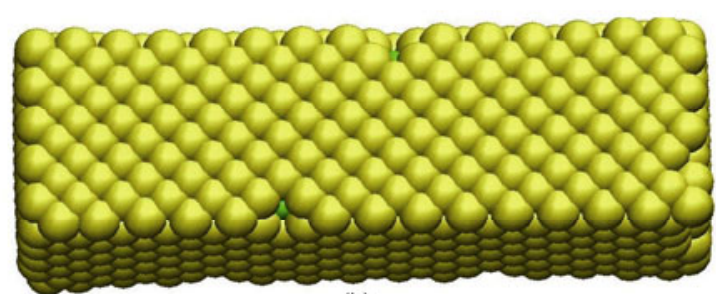

(b)

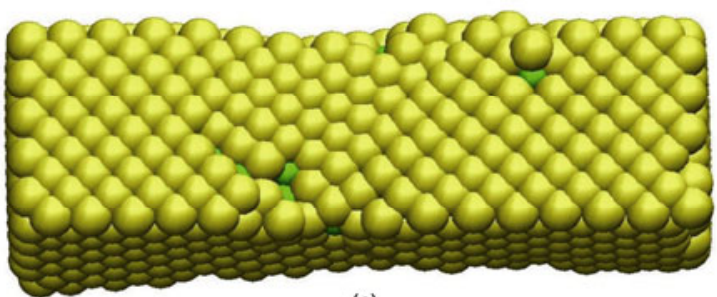

(c)

Figure 9 Vacancy immigration and surface defect induced dislocation nucleation (atomic structures corresponding to various strain levels as indicated in Figure 5) with NW size $a=18.1$ Å. (a) Initial smooth atomic surface; (b) defects immigrate to the surface; (c) yielding induced by surface defect.

scatter data appear in small scales, however the data tend to converge in large scale [16]. The standard deviation (Std) is calculated on each size of NW by using 500 to 1000 data points from MD. The Std is expressed by

$$
\operatorname{Std}=\sqrt{\frac{1}{N} \sum_{i=1}^{N}\left(\sigma_{y}-\bar{\sigma}_{y}\right)},
$$

where $N$ is the sample numbers at a certain size of NW with a specific $c_{v}$, and $\bar{\sigma}_{y}$ is the convergent yield stress at a certain size of NW with a specific $c_{v}$. We study the averaged Std over $c_{v}=0.1 \%$ to $c_{v}=1 \%$ as the change of NW size.

Figure 10 shows Std dependence on the surface volume ratio $\boldsymbol{r}$. It clearly indicates that in small scale with high $\boldsymbol{r}$, the measured yield value may be scattered as much as $0.9 \mathrm{GPa}$. The dispersion goes down as $\boldsymbol{r}$ decreases. As seen from previous discussion, the interaction between vacancy and sur- face becomes intensive as $\boldsymbol{r}$ reaches a maximum value. This triggers a high dispersion of yielding value of NWs. It should be noted that in this figure, the different realizations show almost the same value of Std. The relation between Std and $\boldsymbol{r}$ can be fitted with a linear function as: $\sigma_{y}=$ $0.9747 r+0.1093$, where the dimensions of $\sigma_{y}$ and $r$ are GPa and $\AA^{-1}$, respectively.

\section{Conclusion}

In summary, the effect of vacancy on the tensile yield stress of NW has been studied using MD simulations. The results have shown that by increasing the concentration of vacancy in NW will lead to larger deviations from the theoretical yield point. The variation follows a power law relationship. This contributes to the large gap of yield stress of the NW among experimental, theoretical and simulation results. In 


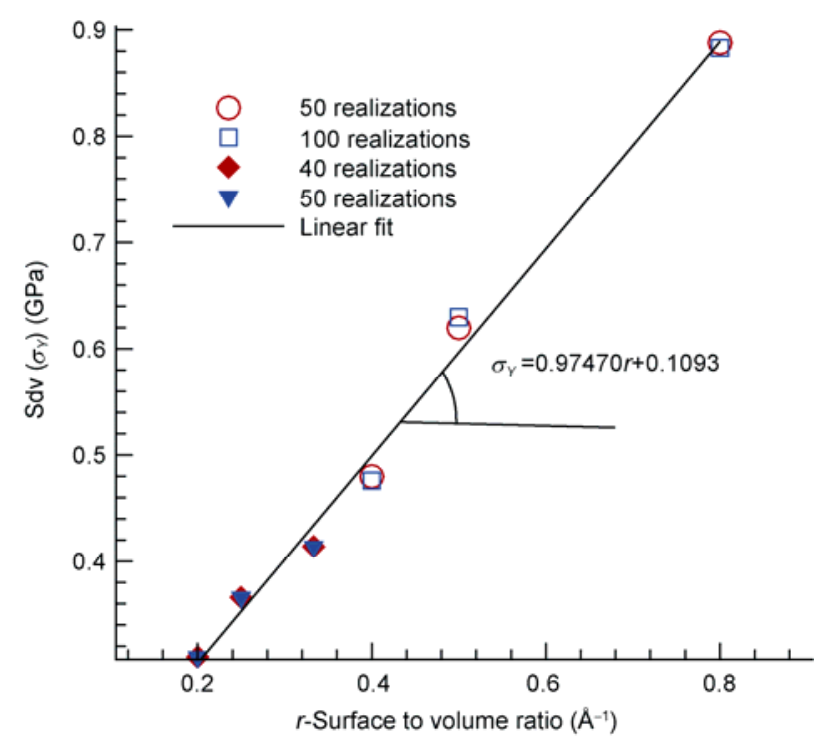

Figure 10 (Color online) Averaged Std (standard deviation) versus $\boldsymbol{r}$ (surface to volume ratio).

the experimental measurement, the large amount of imperfection comes from the manufacturing process which strongly affects the mechanical properties of NW.

Size effect of yield behavior has also been observed. Surface volume ratio is a key factor which affects the scattering of measured values. Yield behavior of small NW is more sensitive to the presence of initial vacancies. This may be because surface stress always appear on small scale material as a result of large specific surface area (surface to volume ratio), leading to defect starvation by immigrating to the free surface. Surface defect induced dislocation nucleation also occurs after the vacancy reaches surface, and this leads to lower yield strength. From experiments [16-18], the dispersion of yield strength is large at small scale which is consistent with our findings.

Deformation mechanism in NW is connected with size, shape and crystal orientation of the NW [47,48], and it should be valuable for further studies. Dislocation nucleation is also strong temperature-dependence [12], and vacancies interactions are related to the thermal fluctuations, and they are also valuable for further investigations in the future.

This work was supported by the National Natural Science Foundation of China (Grants Nos. 50904071, 11021262, 10932011 and 91116003), the Fundamental Research Funds for the Central Universities (Grant No. 2010QZ01), and the National Basic Research Program of China (Grant No. 2012CB937500). LIU XiaoMing acknowledges the using of the super cluster millipede for large scale computations from Groningen University, the Netherlands. We acknowledge anonymous reviewers for constructive advice and language revision.

1 Agrawal R, Peng B, Gdoutos E E, et al. Elasticity size effects in ZnO nanowires- A combined experimental computational approach. Nano
Lett, 2008, 8: 3668-3674

2 Liu X M, Liu Z L, You X C, et al. The stability of Fcc crystal cu under uniaxial loading in [001] direction. Mod Phys Lett B, 2009, 23: 1871-1880

3 Wu B, Heidelberg A, Boland J J. Mechanical properties of ultrahigh-strength gold nanowires. Nat Mater, 2005, 4: 525-529

4 Ogata S, Li J, Yip S. Ideal pure shear strength of aluminum and copper. Science, 2002, 298: 807-811

5 Marszalek P E, Greenleaf W J, Li H, et al. Atomic force microscopy captures quantized plastic deformation in gold nanowires. Proc Nat Acad Sci USA, 2000, 97: 6282-6287

6 Ji C, Park H S. Geometric effects on the inelastic deformation of metal nanowires. Appl Phys Lett, 2006, 89: 181916

7 Park H S, Gall K, Zimmerman J A. Deformation of FCC nanowires by twinning and slip. J Mech Phys Solids, 2006, 54: 1862-1881

8 Koh S J A, Lee H P, Lu C, et al. Molecular dynamics simulation of a solid platinum nanowire under uniaxial tensile strain: Temperature and strain-rate effects. Phys Rev B, 2005, 72: 085414

9 Koh S J A, Lee H P. Molecular dynamics simulation of size and strain rate dependent mechanical response of FCC metallic nanowires. Nanotech, 2006, 17: 3451-3561

10 Diao J, Gall K, Dunn M L. Yield strength asymmetry in metal nanowires. Nano Lett, 2004, 4: 1863-1867

11 Liang W, Zhou M, Ke F. Shape memory effect in $\mathrm{Cu}$ nanowires. Nano Lett, 2005, 5: 2039-2043

12 Zhu T, Li J, Samanta A, et al. Temperature and strain-rate dependence of surface dislocation nucleation. Phys Rev Lett, 2008, 100: 025502

13 Cao A, Ma E. Sample shape and temperature strongly influence the yield strength of metallic nanopillars. Acta Mater, 2008, 56: 4816-4828

14 Mastorakos I N, Zbib H M, Bahr D F, et al. Pseudoelastic behavior of $\mathrm{Cu}-\mathrm{Ni}$ composite nanowires. Appl Phys Lett, 2009, 94: 043104

15 Liu S Y, Soh A K, Hong L. Lateral surface induced effects on ultra-thin amorphous Co nanowire. J Phys D-Appl Phys, 2009, 42: 215002

16 Dou R, Derby B. The strength of gold nanowire forests. Scripta Mater, 2008, 59: 151-154

17 Greer J R, Oliver W C, Nix W D. Size dependence of mechanical properties of gold at the micron scale in the absence of strain gradients. Acta Mater, 2005, 53: 1821-1830

18 Volkert C A, Lilleodden E T, Kramer D, et al. Approaching the theoretical strength in nanoporous Au. Appl Phys Lett, 2006, 89: 061920

19 Cao A, Wei Y. Atomistic simulations of the mechanical behavior of fivefold twinned nanowires. Phys Rev B, 2006, 74: 214108

$20 \mathrm{Wu}$ B, Heidelberg A, Boland J J, et al. Microstructure-hardened silver nanowires. Nano Lett, 2006, 6: 468-473

21 Park H S, Cai W, Espinosa H D, et al. Mechanics of crystalline nanowires. MRS Bull, 2009, 34: 178-183

22 Pastor-Abia L, Caturla M J, SanFabian E, et al. Abnormal stress drop at the yield point of aluminum nanowires: A molecular dynamics study. Phys Rev B, 2011, 83: 165441

23 Shan Z W, Mishra R K, Asif S A S, et al. Mechanical annealing and source-limited deformation in submicrometre-diameter Ni crystals. Nat Mater, 2007, 7: 115-119

24 Sadeghian H, Goosen H, Bossche A, et al. On the size-dependent elasticity of silicon nanocantilevers: Impact of defects. J Phys D-Appl Phys, 2011, 44: 072001

25 Sadeghian H, Yang C K, Goosen J F L, et al. Effects of size and defects on the elasticity of silicon nanocantilevers. J Micromech Microeng, 2010, 20: 064012

26 Njeim E K, Bahr D F. Atomistic simulations of nanoindentation in the presence of vacancies. Scripta Mater, 2010, 62: 598-591

27 Cao A J, Wei Y G, Mao S X. Deformation mechanisms of face-cen- 
tered-cubic metal nanowires with twin boundaries. Appl Phys Lett, 2007, 90: 151909

28 Yang Z, Lu Z, Zhao Y P. Atomistic simulation on size-dependent yield strength and defects evolution of metal nanowires. Computat Mater Sci, 2009, 46: 142-148

29 Mishin Y, Mehl M J, Papaconstantopoulos D A, et al. Structural stability and lattice defects in copper: $\mathrm{Ab}$ initio, tight-binding, and embedded-atom calculations. Phys Rev B, 2001, 63: 224106

30 Nosé S. A unified formulation of the constant temperature molecular dynamics methods. J Chem Phys, 1984, 81: 511-517

31 Li J, Van Vliet K J, Zhu T, et al. Atomistic mechanisms governing elastic limit and incipient plasticity in crystals. Nature, 2002, 418: 307-309

32 Plimpton S. Fast parallel algorithms for short-range molecular dynamics. J Comput Phys, 1995, 117: 1-19

33 Honeycutt J D, Andersen H C. Molecular dynamics study of melting and freezing of small Lennard-Jones clusters. J Phys Chem, 1987, 91: 4950-4955

34 Humphrey W, Dalke A, Schulten K. VMD: Visual molecular dynamics. J Molecular Graph, 1996, 14: 33-39

35 Ma X L, Yang W. Supersonic wave propagation in $\mathrm{Cu}$ under high speed cluster impact. Nanotechnology 2004, 15: 449-454

36 Li X, Wei Y, Lu L, et al. Dislocation nucleation governed softening and maximum strength in nano-twinned metals. Nature, 2010, 464: 877-881

37 Liu X M, You X C, Liu Z L, et al. Atomistic simulations of tension properties for bi-crystal copper with twist grain boundary. J Phys D-Appl Phys, 2009, 42: 035404

38 Wang D, Zhao J, Hu S, et al. Where, and how, does a nanowire break? Nano Lett, 2007, 7: 1208-1212
39 Liu Y, Zhao J. The size dependence of the mechanical properties and breaking behavior of metallic nanowires: A statistical description. Comput Mater Sci, 2010, 50: 1418-1423

40 Liu Y, Zhao J, Wang F. Influence of length on shock-induced breaking behavior of copper nanowires. Phys Rev B, 2009, 80: 115417

41 Kinoshita C, Eguchi T. Vacancy concentration and arrangement of atoms and vacancies in metals and alloys. Acta Metall, 1972, 20: 45-54

42 Luo S N, Germann T C, Tonks D L. The effect of vacancies on dynamic response of single crystal $\mathrm{Cu}$ to shock waves. J Appl Phys 2010, 107: 056102

43 Huang P H, Fang T H, Chou C S. The coupled effects of size, shape, and location of vacancy clusters on the structural deformation and mechanical strength of defective nanowires. Curr Appl Phys, 2011, 11: 878-891

44 Diao J, Gall K, Dunn M L. Surface-stress-induced phase transformation in metal nanowires. Nat Mater, 2003, 2: 656-660

45 Nix W D, Greer J R, Feng G, et al. Deformation at the nanometer and micrometer length scales: Effects of strain gradients and dislocation starvation. Thin Solid Films, 2007, 515: 3152-3159

46 Kolluri K, Gungor M R, Maroudas D. Molecular-dynamics simulations of stacking-fault-induced dislocation annihilation in prestrained ultrathin single-crystalline copper films. J Appl Phys, 2009, 105: 093515

47 Sørensen M R, Brandbyge M, Jacobsen K W. Mechanical deformation of atomic-scale metallic contacts: Structure and mechanisms. Phys Rev B, 1998, 57: 3283-3294

48 Ji C, Park H S. The coupled effects of geometry and surface orientation on the mechanical properties of metal nanowires. Nanotechnology, 2007, 18: 305704 